\title{
An Invariant Property of Balls in Arrangements of Hyperplanes*
}

\author{
Boris Aronov, ${ }^{1}$ Daniel Q. Naiman, ${ }^{2}$ János Pach, ${ }^{3,4}$ and Micha Sharir ${ }^{4,5}$ \\ ${ }^{1}$ Department of Computer Science, Polytechnic University, \\ Brooklyn, NY 11201, USA \\ ${ }^{2}$ Department of Mathematical Sciences, The Johns Hopkins University, \\ Baltimore, MD 21218, USA \\ ${ }^{3}$ Mathematical Institute of the Hungarian Academy of Sciences, \\ POB 127, H-1354 Budapest, Hungary \\ ${ }^{4}$ Courant Institute of Mathematical Sciences, New York University, \\ New York, NY 10012, USA \\ ${ }^{5}$ School of Mathematical Sciences, Tel-Aviv University, \\ Tel-Aviv 69978, Israel
}

\begin{abstract}
Let $\mathscr{H}$ be a collection of $n$ hyperplanes in $d$-space in general position. For each tuple of $d+1$ hyperplanes of $\mathscr{H}$ consider the open ball inscribed in the simplex that they form. Let $\mathscr{B}_{k}$ denote the number of such balls intersected by exactly $k$ hyperplanes, for $k=0,1, \ldots, n-d-1$. We show that
\end{abstract}

$$
\left|\mathscr{Q}_{k}\right|=\left(\begin{array}{c}
n-k-1 \\
d
\end{array}\right) \text {. }
$$

The result stated in the abstract has been recently obtained by Naiman [N] using a different and more involved proof technique. We present here a short, simple, and purely combinatorial proof.

Let $\mathscr{H}$ be a collection of $n$ hyperplanes in $d$-space in general position. By this

\footnotetext{
* Work on this paper by the second author has been supported by National Science Foundation Grant DMS-91-03126. Work by the third and fourth authors has been supported by National Science Foundation Grant CCR-89-01484. Work by the third author has also been supported by Hungarian Science Foundation Grant OTKA-1814. Work by the fourth author has also been supported by Office of Naval Research Grant N00014-90-J-1284, and by grants from the U.S.-Israeli Binational Science Foundation, the G.I.F.- the German Israeli Foundation for Scientific Research and Development, and the Fund for Basic Research administered by the Israeli Academy of Sciences.
} 
we mean that any $d$ of these hyperplanes meet in exactly one point, the intersection of any $d+1$ of them is empty, and no $d+2$ of them are tangent to a common sphere. Let $\mathscr{A}(\mathscr{H})$ denote the arrangement of $\mathscr{H}$, namely, the cell decomposition of $d$-space induced by the hyperplanes of $\mathscr{H}$ (see [E] for more details concerning arrangements). For each $(d+1)$-tuple $\tau$ of distinct hyperplanes of $\mathscr{H}$, let $B_{\tau}$ denote the open ball inscribed in the simplex formed by the hyperplanes of $\tau$. Let $\mathscr{B}=\mathscr{B}(\mathscr{H})$ denote the resulting collection of $\left({ }_{d}+{ }_{1}\right)$ balls, and for each $k=$ $0,1, \ldots, n-d-1$, let $\mathscr{B}_{k}=\mathscr{B}_{k}(\mathscr{H})$ denote the set of balls of $\mathscr{B}$ which are met by exactly $k$ hyperplanes of $\mathscr{H}$.

Theorem 1. For each $k=0, \ldots, n-d-1$ we have

$$
\left|\mathscr{B}_{k}\right|=\left(\begin{array}{c}
n-k-1 \\
d
\end{array}\right) .
$$

Proof. The proof relies on the following simple lemma:

Lemma 2. For any set $\mathscr{H}$ of hyperplanes in general position in $d$-space, $\left|\mathscr{B}_{0}(\mathscr{H})\right|$ is equal to the number of bounded cells of $\mathscr{A}(\mathscr{H})$.

Proof. If $B \in \mathscr{B}_{0}(\mathscr{H})$, then $B$ is fully contained within some cell of $\mathscr{A}(\mathscr{H})$. Since $B$ is also contained in a simplex formed by some $d+1$ hyperplanes of $\mathscr{H}$, it follows that this cell must be bounded. We claim that each bounded cell contains exactly one ball of $\mathscr{B}_{0}(\mathscr{H})$. Indeed, let $C$ be a bounded cell of $\mathscr{A}(\mathscr{H})$, and let $B$ be the largest ball inscribed in $C$. The general position assumption is easily seen to imply that $B$ is tangent to exactly $d+1$ hyperplanes of $\mathscr{H}$, and straightforward analysis shows that $B$ must therefore be the ball inscribed in the simplex $S$ formed by these hyperplanes. This implies that $B \in \mathscr{B}_{0}(\mathscr{H})$. Assume, for the sake of contradiction, that $C$ contains another ball $B^{\prime} \in \mathscr{B}_{0}(\mathscr{H})$. Obviously, $\operatorname{conv}\left\{B \cup B^{\prime}\right\} \subset C$. Thus $B^{\prime}$ can be translated within $C$ toward the center of $B$. This contradicts the fact that $B^{\prime}$ is inscribed in a simplex determined by $d+1$ hyperplanes belonging to $\mathscr{H}$.

\section{Corollary 3.}

$$
\left|\mathscr{B}_{0}(\mathscr{H})\right|=\left(\begin{array}{c}
|\mathscr{H}|-1 \\
d
\end{array}\right)
$$

Proof. The number of bounded cells in an arrangement of a set $\mathscr{H}$ of hyperplanes in general position in $d$-space is $\left(\mathscr{*}_{d} \mid-1\right)$ [G], [Z].

Now we return to the proof of the theorem. We use induction on $k$. The base case $k=0$ has been taken care of in the preceding corollary. Suppose the claim is true for all $k^{\prime}<k$. We are going to count in two different ways the number $N$ of pairs $(\mathscr{G}, B)$, where $\mathscr{G}$ is a subset of $\mathscr{H}$ of size $k$ and $B \in \mathscr{B}{ }_{0}(\mathscr{H} \backslash \mathscr{G})$. 
By Corollary 3, we have

$$
N=\left(\begin{array}{l}
n \\
k
\end{array}\right)\left(\begin{array}{c}
n-k-1 \\
d
\end{array}\right)
$$

On the other hand, let $(\mathscr{G}, B)$ be such a pair. Then $B$ must belong to $\mathscr{B}_{j}(\mathscr{H})$, for some $j=0,1, \ldots, k$, and $\mathscr{G}$ must consist of the $j$ hyperplanes intersecting $B$, plus $k-j$ other elements chosen from the set of $n-d-1-j$ hyperplanes that neither are tangent to nor cross $B$. This easily implies

$$
N=\sum_{j=0}^{k}\left(\begin{array}{c}
n-d-j-1 \\
k-j
\end{array}\right)\left|\mathscr{B}_{j}(\mathscr{H})\right|=\left|\mathscr{B}_{k}(\mathscr{H})\right|+\sum_{j=0}^{k-1}\left(\begin{array}{c}
n-d-j-1 \\
k-j
\end{array}\right)\left|\mathscr{B}_{j}(\mathscr{H})\right|
$$

Hence, by the induction hypothesis,

$$
\left|\mathscr{B}_{k}(\mathscr{H})\right|=\left(\begin{array}{l}
n \\
k
\end{array}\right)\left(\begin{array}{c}
n-k-1 \\
d
\end{array}\right)-\sum_{j=0}^{k-1}\left(\begin{array}{c}
n-d-j-1 \\
k-j
\end{array}\right)\left(\begin{array}{c}
n-j-1 \\
d
\end{array}\right)
$$

or, by standard manipulation of binomial coefficients,

$$
\begin{aligned}
\left|\mathscr{B}_{k}(\mathscr{H})\right| & =\left(\begin{array}{l}
n \\
k
\end{array}\right)\left(\begin{array}{c}
n-k-1 \\
d
\end{array}\right)-\left(\begin{array}{c}
n-k-1 \\
d
\end{array}\right) \cdot \sum_{j=0}^{k-1}\left(\begin{array}{c}
n-j-1 \\
k-j
\end{array}\right) \\
& =\left(\begin{array}{c}
n-k-1 \\
d
\end{array}\right) \cdot\left[\left(\begin{array}{l}
n \\
k
\end{array}\right)-\sum_{q=1}^{k}\left(\begin{array}{c}
n-k-1+q \\
q
\end{array}\right)\right] \\
& =\left(\begin{array}{c}
n-k-1 \\
d
\end{array}\right) \cdot\left[\left(\begin{array}{l}
n \\
k
\end{array}\right)-\left(\left(\begin{array}{l}
n \\
k
\end{array}\right)-1\right)\right] \\
& =\left(\begin{array}{c}
n-k-1 \\
d
\end{array}\right)
\end{aligned}
$$

(see, e.g., p. 174 of [GKP]). Hence the claim holds for $k$ too, and this completes the proof of the theorem.

Remarks. (1) The above recurrence for $\left|\mathscr{B}_{k}(\mathscr{H})\right|$ can also be derived by applying the random sampling technique of Clarkson and Shor [CS].

(2) Lemma 2 also holds for homothetic copies of any fixed open convex body $K$. Thus, with an appropriately modified notion of general position, we obtain 
that the number of $(d+1)$-tuples in $\mathscr{H}$ that determine a simplex $S$ such that the largest homothet of $K$ inscribed in $S$ intersects exactly $k$ elements of $\mathscr{H}$ is

$$
\left(\begin{array}{c}
n-k-1 \\
d
\end{array}\right)
$$

(3) A more geometric approach to proving the invariance of $\left|\mathscr{B}_{k}(\mathscr{H})\right|$ was taken in [N]: One considers the space of all $n$-tuples of hyperplanes in $d$-space not containing the origin, with a natural differentiable structure that it inherits as a product of copies of $S^{d-1} \times(0, \infty)$, and observes that the set of tuples of hyperplanes which are not in general position can be viewed as a union of submanifolds of codimension at least one. To prove invariance, it is thus sufficient to show that a smooth motion of the hyperplanes that only encounters degeneracies of codimension one maintains the invariant across each such degeneracy. The latter statement is then proven by explicit enumeration of all possible degeneracies of codimension one. An alternative geometric proof begins by arguing that translating a hyperplane parallel to itself maintains the invariant across degeneracies. The argument is completed by an easy explicit computation of $\left|\mathscr{B}_{k}(\mathscr{H})\right|$ for any set $\mathscr{H}$ of hyperplanes in general position, in which orientations of the hyperplanes are prescribed, but one is free to choose their relative positions.

(4) We also mention a recent related result of Clarkson [C] that can be proven using methods employed in this paper. He shows that the number of local maxima on the $k$-level of an arrangement of $n$ hyperplanes in general position in $d$-space is at most

$$
\min \left\{\left(\begin{array}{c}
k+d-1 \\
d-1
\end{array}\right),\left(\begin{array}{c}
n-k-1 \\
d-1
\end{array}\right)\right\}
$$

Here the $k$-level is the boundary of the set of all points that have no more than $k$ hyperplanes directly below them. Though seemingly unrelated, this quantity is in fact a generalization of $\left|\mathscr{B}_{k}\right|$. Namely, consider a set $\mathscr{H}$ of $n$ hyperplanes in $d$-space. We construct a set $\mathscr{H}^{\prime}$ of $2 n+1$ hyperplanes in $(d+1)$-space as follows: Identify $d$-space with the hyperplane $h_{0}$ defined by $x_{d+1}=0$. For each $h \in \mathscr{H}$, let $h^{\prime}, h^{\prime \prime}$ be the two hyperplanes in $(d+1)$-space passing through $h$ at $45^{\circ}$ with $h_{0}$. $\mathscr{H}^{\prime}$ consists of $h_{0}$ and of the hyperplanes $h^{\prime}, h^{\prime \prime}$ corresponding to each hyperplane $h \in \mathscr{H}$. It is easily checked that local maxima of the $(n+1+k)$-level in $\mathscr{A}^{\prime}=\mathscr{A}\left(\mathscr{H}^{\prime}\right)$ stand in one-to-one correspondence with balls of $\mathscr{B}_{k}(\mathscr{H})$. For example, the $n$-level in $\mathscr{A}^{\prime}$ is just the hyperplane $h_{0}$. The $(n+1)$-level is the union of all facets of $\mathscr{A}^{\prime}$ lying just above $h_{0}$. The number of local maxima there is exactly the number of bounded cells of $\mathscr{A}^{\prime}$ lying above $h_{0}$ and touching it in a $d$-face, which is equal to the number of bounded cells of $\mathscr{A}(\mathscr{H})$, which in turn is equal to $\left|\mathscr{B}_{0}(\mathscr{H})\right|$, by Lemma 2. The case $k \geq 1$ is treated similarly. It is now easily checked that Clarkson's bound, applied to $\mathscr{A}^{\prime}$, yields an upper bound on $\left|\mathscr{B}_{k}(\mathscr{H})\right|$, which is the same as the (exact) bound given in Theorem 1. 


\section{References}

[C] K. L. Clarkson, A bound on local minima of arrangements that implies the upper bound theorem, Discrete Comput. Geom., this issue, 427-433.

[CS] K. Clarkson and P. Shor, Applications of random sampling in computational geometry, II, Discrete Comput. Geom. 4 (1989), 387-421.

[E] H. Edelsbrunner, Algorithms in Combinatorial Geometry, Springer-Verlag, Heidelberg, 1987.

[GKP] R. Graham, D. Knuth, and O. Patashnik, Concrete Mathematics, Addison-Wesley, Reading, MA, 1989.

[G] B. Grünbaum, Convex Polytopes, Wiley, New York, 1967.

[N] D. Q. Naiman, Hyperplane arrangements and related invariants, Unpublished manuscript, January 1992.

[Z] T. Zaslavsky, Facing Up to Arrangements: Face Count Formulas for Partitions of Space by Hyperplanes. Memoirs of the American Mathematical Society (1), Vol. 154, American Mathematical Society, Providence, RI, 1975.

Received February 3, 1992, and in revised form June 22, 1992, and August 17, 1992. 